\title{
The expected values of Sombor indices in random hexagonal chains, phenylene chains and Sombor indices of some chemical graphs *
}

\author{
Xiaona Fang, Lihua You`, Hechao Liu \\ School of Mathematical Sciences, South China Normal University, \\ Guangzhou, 510631, P. R. China
}

\begin{abstract}
Hexagonal chains are a special class of catacondensed benzenoid system and phenylene chains are a class of polycyclic aromatic compounds. Recently, A family of Sombor indices was introduced by Gutman in the chemical graph theory. It had been examined that these indices may be successfully applied on modeling thermodynamic properties of compounds. In this paper, we study the expected values of the Sombor indices in random hexagonal chains, phenylene chains, and consider the Sombor indices of some chemical graphs such as graphene, coronoid systems and carbon nanocones.
\end{abstract}

Keywords: Sombor index; random hexagonal chain; random phenylene chain; chemical graph; expected value.

\section{Introduction}

Chemical graph theory is an interdisciplinary field of science which relates chemistry with a branch of mathematical modeling of graphs. Topological indices are graph invariants that play an important role in chemical and pharmaceutical sciences, since they can be used to predict physicochemical properties of organic compounds [34]. There are lots of topological indices in the literature of chemical graph theory. Recently, Gutman introduces a family of Sombor indices in the chemical graph theory [14]. It was examined in [31] that the Sombor index, reduced Sombor index and average Sombor index showed satisfactory predictive and discriminative potential in modeling entropy and enthalpy of vaporization of alkanes. The results of testing predictive potential of Sombor indices indicate that these descriptors may be successfully applied on modeling thermodynamic properties of compounds.

Let $G=(V, E)$ be a finite, connected, simple graph with vertex set $V=V(G)$ and edge set $E=E(G)$, where $|V(G)|$ is the number of vertices and $|E(G)|$ is the number of edges. We

\footnotetext{
${ }^{*}$ This work is supported by the National Natural Science Foundation of China (Grant No. 11971180), the Guangdong Provincial Natural Science Foundation (Grant No. 2019A1515012052).

†Corresponding author: ylhua@scnu.edu.cn
} 
denote the degree of a vertex $i$ in $G$ by $d_{i}$. The (ordinary) Sombor index is defined as

$$
S O(G)=\sum_{i \sim j} \sqrt{d_{i}^{2}+d_{j}^{2}}
$$

the reduced Sombor index is defined as

$$
S O_{r e d}(G)=\sum_{i \sim j} \sqrt{\left(d_{i}-1\right)^{2}+\left(d_{j}-1\right)^{2}}
$$

and the average Sombor index, as

$$
S O_{a v r}(G)=\sum_{i \sim j} \sqrt{\left(d_{i}-\bar{d}\right)^{2}+\left(d_{j}-\bar{d}\right)^{2}},
$$

where $\bar{d}=\frac{2 \cdot|E(G)|}{|V(G)|}$ is the average degree of graph $G \sqrt{14}$. In this paper, Sombor indices refer to Sombor index, reduced Sombor index and average Sombor index. Let $a$ be any real number or parameter of graph $G$. We generalize the Sombor indices with $a$. The generalized index is defined as

$$
S O_{a}(G)=\sum_{i \sim j} \sqrt{\left(d_{i}-a\right)^{2}+\left(d_{j}-a\right)^{2}} .
$$

It's clear when $a=0, S O_{a}(G)=S O(G)$, when $a=1, S O_{a}(G)=S O_{\text {red }}(G)$ and when $a=\bar{d}$, $S O_{a}(G)=S O_{a v r}(G)$.

Sombor indices have attracted much attention due to good chemical applicability. Cruz, Gutman and Rada characterized the extremal graphs of the chemical graphs, chemical trees and hexagon systems with respect to Sombor index [7]. In [4], the Sombor index of polymer graphs which can be decomposed into monomer units was considered. In [8], the extremal values of the Sombor index in unicyclic and bicyclic graphs were studied. Das, Cevik, Cangul and Shang presented lower and upper bounds on the Sombor index of graphs by using some graph parameters and obtain several relations on Sombor index with the first and second Zagreb indices of graphs [10]. More results of Sombor indices can be found in $11,12,14,16,19,22,24$, $31,32,35$. In Section 2, we study the expected values of the Sombor indices in the random hexagonal chains and random phenylene chains, and make a comparison between the expected values. In Section 3, we study the Sombor indices of some graphs that are of importance in chemistry such as graphene, coronoid systems and carbon nanocones, and give numerical comparison of the Sombor indices and graphical profiles of the comparison. 


\section{The expected values of Sombor indices in random hexag- onal chains and phenylene chains}

Random molecular graphs are of great importance for theoretical chemistry. There are many results about the extremal values of topological indices of random molecular graphs in recent years $17,23,27,28,36$. In this section, we study the expected values of the Sombor indices in random hexagonal chains and phenylene chains.

We say an edge is $(i, j)$-type if it joins a vertex with degree $i$ and a vertex with degree $j$ in $G$. Let $m_{i j}(G)$ be the number of edges of $(i, j)$-type. Then we have the following Proposition.

Proposition 2.1. Let $G$ be a graph. If there exists only $(2,2),(2,3)$ and $(3,3)$-type of edges in $G$, then we have

$$
S O_{a}(G)=\sqrt{2} \cdot|2-a| \cdot m_{22}(G)+\sqrt{2 a^{2}-10 a+13} \cdot m_{23}(G)+\sqrt{2} \cdot|3-a| \cdot m_{33}(G) .
$$

Proof. Since there exists only $(2,2),(2,3)$ and (3,3)-type of edges in $G$, by (1), we have

$$
\begin{aligned}
S O_{a}(G) & =\sqrt{(2-a)^{2}+(2-a)^{2}} \cdot m_{22}(G)+\sqrt{(2-a)^{2}+(3-a)^{2}} \cdot m_{23}(G) \\
& +\sqrt{(3-a)^{2}+(3-a)^{2}} \cdot m_{33}(G) \\
& =\sqrt{2} \cdot|2-a| \cdot m_{22}(G)+\sqrt{2 a^{2}-10 a+13} \cdot m_{23}(G)+\sqrt{2} \cdot|3-a| \cdot m_{33}(G) .
\end{aligned}
$$

The proof is completed.

\subsection{Random hexagonal chains}

A benzenoid system is a finite connected subgraph of the infinite hexagonal lattice without cut vertices or non-hexagonal interior faces. A benzenoid system without any hexagon which has more than two neighboring hexagons is called a hexagonal chain, denoted by $H X G_{n}$. For $n \geq 3$, the terminal hexagon can be attached in three ways, which results in the local arrangements, we describe as $H X G_{n}^{1}, H X G_{n}^{2}$, and $H X G_{n}^{3}$, respectively, see Figure 1.

A random hexagonal chain $H X G\left(n ; p_{1}, p_{2}\right)$ with $n$ hexagons is a hexagonal chain obtained by stepwise addition of terminal hexagons. At each step $t(=3,4, \cdots, n)$, a random selection is made from one of the three possible constructions:

(1) $H X G_{t-1} \rightarrow H X G_{t}^{1}$ with probability $p_{1}$;

(2) $H X G_{t-1} \rightarrow H X G_{t}^{2}$ with probability $p_{2}$;

(3) $H X G_{t-1} \rightarrow H X G_{t}^{3}$ with probability $1-p_{1}-p_{2}$, where $p_{1}, p_{2}$ are constants, irrelative to the step parameter $t$. 

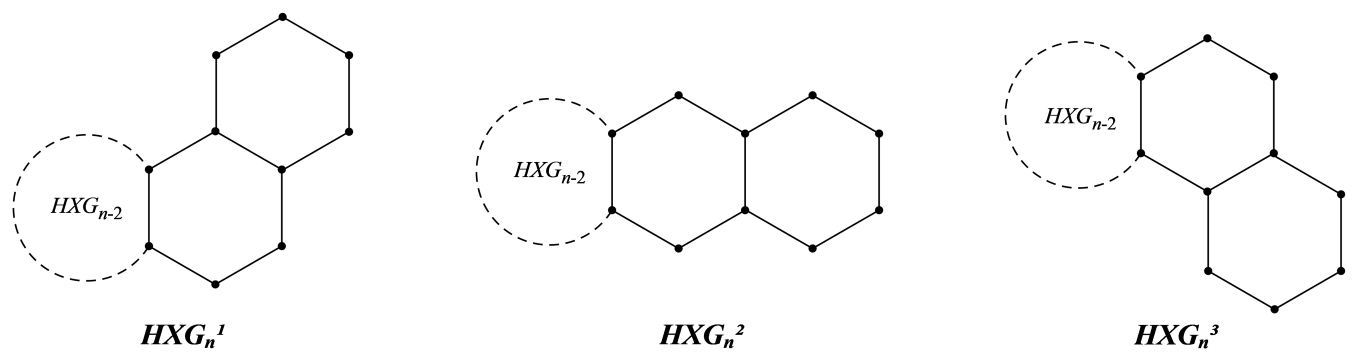

Figure 1: The three types of local arrangements in the hexagonal chains.

Since $H X G\left(n ; p_{1}, p_{2}\right)$ is a random hexagonal chain, $S O\left(H X G\left(n ; p_{1}, p_{2}\right)\right), S O_{\text {red }}\left(H X G\left(n ; p_{1}, p_{2}\right)\right)$ and $S O_{a v r}\left(H X G\left(n ; p_{1}, p_{2}\right)\right)$ are random variables. We denote the expected values of these indices by $E_{n}^{a}=E\left[S O_{a}\left(H X G\left(n ; p_{1}, p_{2}\right)\right)\right]$. When $a=0, E_{n}^{a}=E_{n}$, when $a=1, E_{n}^{a}=E_{n}^{r e d}$ and when $a=\bar{d}, E_{n}^{a}=E_{n}^{a v r}$. In this section, $a$ is a constant.

Theorem 2.2. Let $H X G\left(n ; p_{1}, p_{2}\right)$ be the hexagonal chain of length $n(\geq 2)$. Then

$$
\begin{gathered}
E_{n}^{a}=2\left(n p_{2}-2 p_{2}+n\right) \sqrt{2 a^{2}-10 a+13}+\sqrt{2}\left(-n p_{2}+2 p_{2}+n+4\right) \cdot|2-a| \\
+\sqrt{2}\left(-n p_{2}+2 p_{2}+2 n-3\right) \cdot|3-a|, \\
E_{n}=\left[(2 \sqrt{13}-5 \sqrt{2}) p_{2}+8 \sqrt{2}+2 \sqrt{13}\right] n+(10 \sqrt{2}-4 \sqrt{13}) p_{2}-\sqrt{2}, \\
E_{n}^{r e d}=\left[(2 \sqrt{5}-3 \sqrt{2}) p_{2}+5 \sqrt{2}+2 \sqrt{5}\right] n+(6 \sqrt{2}-4 \sqrt{5}) p_{2}-2 \sqrt{2}, \\
E_{n}^{a v r}=\left[2\left(p_{2}+1\right) \sqrt{2 \bar{d}^{2}-10 \bar{d}+13}+\sqrt{2}\left(4-p_{2}-\bar{d}\right)\right] n \\
-4 p_{2} \sqrt{2 \bar{d}^{2}-10 \bar{d}+13}+\sqrt{2}\left(2 p_{2}+7 \bar{d}-17\right), \text { where } \bar{d}=\frac{5 n+1}{2 n+1} .
\end{gathered}
$$

Proof. From the structure of the hexagonal chain, it is easy to see that there exists only $(2,2)$, $(2,3)$ and $(3,3)$-type of edges. From Proposition 2.1, when $n=2, E_{2}^{a}=4 \sqrt{2 a^{2}-10 a+13}+$ $\sqrt{2}(6 \cdot|2-a|+|3-a|)$.

For $n \geq 3$, there are three possibilities to be considered (see Figure 1).

Case 1. $H X G_{n-1} \rightarrow H X G_{n}^{1}$.

$$
\begin{aligned}
& m_{22}\left(H X G_{n}^{1}\right)=m_{22}\left(H X G_{n-1}\right)+1 ; \\
& m_{23}\left(H X G_{n}^{1}\right)=m_{23}\left(H X G_{n-1}\right)+2 ; \\
& m_{33}\left(H X G_{n}^{1}\right)=m_{33}\left(H X G_{n-1}\right)+2 .
\end{aligned}
$$


Thus, $S O_{a}\left(H X G_{n}^{1}\right)=S O_{a}\left(H X G_{n-1}\right)+2 \sqrt{2 a^{2}-10 a+13}+\sqrt{2}(|2-a|+2 \cdot|3-a|)$.

Case 2. $H X G_{n-1} \rightarrow H X G_{n}^{2}$.

$$
\begin{aligned}
& m_{22}\left(H X G_{n}^{2}\right)=m_{22}\left(H X G_{n-1}\right)+0 ; \\
& m_{23}\left(H X G_{n}^{2}\right)=m_{23}\left(H X G_{n-1}\right)+4 ; \\
& m_{33}\left(H X G_{n}^{2}\right)=m_{33}\left(H X G_{n-1}\right)+1 .
\end{aligned}
$$

Thus, $S O_{a}\left(H X G_{n}^{2}\right)=S O_{a}\left(H X G_{n-1}\right)+4 \sqrt{2 a^{2}-10 a+13}+\sqrt{2} \cdot|3-a|$.

Case 3. $H X G_{n-1} \rightarrow H X G_{n}^{3}$.

$$
\begin{aligned}
& m_{22}\left(H X G_{n}^{3}\right)=m_{22}\left(H X G_{n-1}\right)+1 ; \\
& m_{23}\left(H X G_{n}^{3}\right)=m_{23}\left(H X G_{n-1}\right)+2 ; \\
& m_{33}\left(H X G_{n}^{3}\right)=m_{33}\left(H X G_{n-1}\right)+2 .
\end{aligned}
$$

Thus, $S O_{a}\left(H X G_{n}^{3}\right)=S O_{a}\left(H X G_{n-1}\right)+2 \sqrt{2 a^{2}-10 a+13}+\sqrt{2}(|2-a|+2 \cdot|3-a|)$.

Therefore, $E_{n}^{a}=p_{1} \cdot S O_{a}\left(H X G_{n}^{1}\right)+p_{2} \cdot S O_{a}\left(H X G_{n}^{2}\right)+\left(1-p_{1}-p_{2}\right) \cdot S O_{a}\left(H X G_{n}^{3}\right)=$ $S O_{a}\left(H X G_{n-1}\right)+2\left(p_{2}+1\right) \sqrt{2 a^{2}-10 a+13}+\sqrt{2}\left[\left(1-p_{2}\right) \cdot|2-a|+\left(2-p_{2}\right) \cdot|3-a|\right]$. Since $E\left[E_{n}^{a}\right]=E_{n}^{a}$, we have

$$
E_{n}^{a}=E_{n-1}^{a}+2\left(p_{2}+1\right) \sqrt{2 a^{2}-10 a+13}+\sqrt{2}\left[\left(1-p_{2}\right) \cdot|2-a|+\left(2-p_{2}\right) \cdot|3-a|\right] .
$$

After solving the recurrence relation (7) with initial condition, we get (3).

When $a=0$, we have (4). When $a=1$, we have (5). Since $\left|V\left(H X G\left(n ; p_{1}, p_{2}\right)\right)\right|=4 n+2$, $\left|E\left(H X G\left(n ; p_{1}, p_{2}\right)\right)\right|=5 n+1$, we have $2<\bar{d}=\frac{5 n+1}{2 n+1}<3$. For given $n, \bar{d}$ is a constant and therefore we get (6).

Let $\mathcal{P}_{n}=H X G(n ; 0,1)$ and $\mathcal{R}_{n}=H X G\left(n ; p_{1}, 0\right)$, where $p_{1} \in[0,1]$. By Theorem 2.2, we have

Corollary 2.3. The Sombor indices of $\mathcal{R}_{n}$ and $\mathcal{P}_{n}$ are

$$
\begin{aligned}
& S O_{a}\left(\mathcal{R}_{n}\right)=2 n \sqrt{2 a^{2}-10 a+13}+\sqrt{2}(n+4) \cdot|2-a|+\sqrt{2}(2 n-3) \cdot|3-a|, \\
& S O_{a}\left(\mathcal{P}_{n}\right)=4(n-1) \sqrt{2 a^{2}-10 a+13}+6 \sqrt{2} \cdot|2-a|+\sqrt{2}(n-1) \cdot|3-a| .
\end{aligned}
$$


Corollary 2.4. Among all random hexagonal chains $H X G_{n}(n \geq 2)$, we have

(1) $(8 \sqrt{2}+2 \sqrt{13}) n-\sqrt{2} \leq S O\left(H X G_{n}\right) \leq(3 \sqrt{2}+4 \sqrt{13}) n+9 \sqrt{2}-4 \sqrt{13}$, with left equality iff $G \cong \mathcal{R}_{n}$, right equality iff $G \cong \mathcal{P}_{n}$.

(2) $(5 \sqrt{2}+2 \sqrt{5}) n-2 \sqrt{2} \leq S O_{\text {red }}\left(H X G_{n}\right) \leq(2 \sqrt{2}+4 \sqrt{5}) n+4 \sqrt{2}-4 \sqrt{5}$, with left equality iff $G \cong \mathcal{R}_{n}$, right equality iff $G \cong \mathcal{P}_{n}$.

(3) $2 n \sqrt{2 \bar{d}^{2}-10 \bar{d}+13}+\sqrt{2}[(4-\bar{d}) n+7 \bar{d}-17] \leq S O_{\text {avr }}\left(H X G_{n}\right) \leq 4(n-1) \sqrt{2 \bar{d}^{2}-10 \bar{d}+13}+$ $\sqrt{2}[(3-\bar{d}) n+7 \bar{d}-15]$, where $\bar{d}=\frac{5 n+1}{2 n+1}$, with left equality iff $G \cong \mathcal{R}_{n}$, right equality iff $G \cong \mathcal{P}_{n}$.

Proof. Since $E_{n}=(n-2)(2 \sqrt{13}-5 \sqrt{2}) p_{2}+\sqrt{2}(8 n-1)+2 \sqrt{13} n$ and $(n-2)(2 \sqrt{13}-5 \sqrt{2}) \geq 0$, $S O\left(H X G_{n}\right)$ reaches the maximum value when $p_{2}=1$ and reaches the minimum value when $p_{2}=0$.

Since $E_{n}^{r e d}=(n-2)(2 \sqrt{5}-3 \sqrt{2}) p_{2}+\sqrt{2}(5 n-2)+2 \sqrt{5} n$ and $(n-2)(2 \sqrt{5}-3 \sqrt{2}) \geq 0$, $S O_{\text {red }}\left(H X G_{n}\right)$ reaches the maximum value when $p_{2}=1$ and reaches the minimum value when $p_{2}=0$.

$$
E_{n}^{a v r}=(n-2)\left[2 \sqrt{2 \bar{d}^{2}-10 \bar{d}+13}-\sqrt{2}\right] p_{2}+2 n \sqrt{2 \bar{d}^{2}-10 \bar{d}+13}+\sqrt{2}[(4-\bar{d}) n+7 \bar{d}-17]
$$

can be regarded as a linear function of $p_{2}$. Since $n \geq 2,2 \bar{d}^{2}-10 \bar{d}+13=2\left(\bar{d}-\frac{5}{2}\right)^{2}+\frac{1}{2} \geq \frac{1}{2}$, we have $2 \sqrt{2 \bar{d}^{2}-10 \bar{d}+13}-\sqrt{2} \geq 0$. Thus $S O_{\text {avr }}\left(H X G_{n}\right)$ reaches the maximum value when $p_{2}=1$ and reaches the minimum value when $p_{2}=0$.

Denote by $\mathcal{H C}_{n}$ the set of all hexagonal chains with $n$ hexagons. The average value of Sombor indices among $\mathcal{H C}_{n}$ can be characterized as

$$
A_{a}\left(\mathcal{H C}_{n}\right)=\frac{1}{\left|\mathcal{H C}_{n}\right|} \sum_{G \in \mathcal{H C}_{n}} S O_{a}(G)
$$

Since each element in $\mathcal{H C}_{n}$ has the same probability of occurrence, we have $p_{1}=p_{2}=1-p_{1}-$ $p_{2}=\frac{1}{3}$. Then we have the following theorem.

Theorem 2.5. The average values of Sombor indices among $\mathcal{H C}_{n}$ are

$$
A_{a}\left(\mathcal{H C}_{n}\right)=\frac{4}{3}(2 n-1) \sqrt{2 a^{2}-10 a+13}+\frac{2}{3} \sqrt{2}(n+7) \cdot|2-a|+\frac{\sqrt{2}}{3}(5 n-7) \cdot|3-a| .
$$

\subsection{Random phenylene chains}

The phenylene chains are a class of conjugated hydrocarbons consists of hexagons and squares connected in turn, which has unique physicochemical properties due to their aromatic and antiaromatic rings. In [29,30], Raza studied the expected values of some indices such as sum-connectivity, harmonic, symmetric division, arithmetic bond connectivity and geometric 
indices in random phenylene chains. In the following, we will study the Sombor indices of phenylene chains which are special molecular graphs. A phenylene chain $R P H_{n}$ with $n$ hexagons can be regarded as a phenylene chain $R P H_{n-1}$ with $n-1$ hexagons to which a new terminal hexagon has been adjoined by two edges. For $n \geq 3$, the terminal hexagon can be attached in three ways, which results in the local arrangements, we describe as $R P H_{n}^{1}, R P H_{n}^{2}$, and $R P H_{n}^{3}$, respectively (see Figure 2).

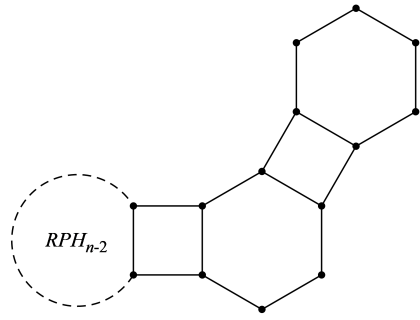

$\mathrm{RPH}_{n}{ }^{1}$

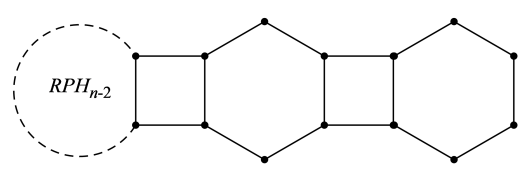

$\mathrm{RPH}_{n}{ }^{2}$

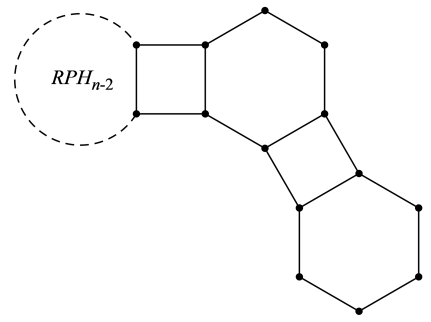

$\mathrm{RPH}_{n}{ }^{3}$

Figure 2: The three types of local arrangements in the random phenylene chains.

A random phenylene chain $R P H\left(n ; p_{1}, p_{2}\right)$ with $n$ hexagons is a polyphenyl chain obtained by stepwise addition of terminal hexagons. At each step $t(=3,4, \cdots, n)$, a random selection is made from one of the three possible constructions:

(1) $R P H_{t-1} \rightarrow R P H_{t}^{1}$ with probability $p_{1}$;

(2) $R P H_{t-1} \rightarrow R P H_{t}^{2}$ with probability $p_{2}$;

(3) $R P H_{t-1} \rightarrow R P H_{t}^{3}$ with probability $1-p_{1}-p_{2}$, where $p_{1}, p_{2}$ are constants, irrelative to the step parameter $t$.

We denote the expected values of Sombor indices by $\mathbb{E}_{n}^{a}=E\left[S O_{a}\left(R P H\left(n ; p_{1}, p_{2}\right)\right)\right], \mathbb{E}_{n}=$ $E\left[S O\left(R P H\left(n ; p_{1}, p_{2}\right)\right)\right], \mathbb{E}_{n}^{r e d}=E\left[S O_{r e d}\left(R P H\left(n ; p_{1}, p_{2}\right)\right)\right]$ and $\mathbb{E}_{n}^{a v r}=E\left[S O_{\text {avr }}\left(R P H\left(n ; p_{1}, p_{2}\right)\right)\right]$. In this section, $a$ is a constant.

Theorem 2.6. Let $\operatorname{RPH}\left(n ; p_{1}, p_{2}\right)$ be the random phenylene chain of length $n(\geq 2)$. Then

$$
\begin{gathered}
\mathbb{E}_{n}^{a}=2\left(n p_{2}-2 p_{2}+n\right) \sqrt{2 a^{2}-10 a+13}+\sqrt{2}\left(-n p_{2}+2 p_{2}+n+4\right) \cdot|2-a| \\
+\sqrt{2}\left(-n p_{2}+2 p_{2}+5 n-6\right) \cdot|3-a|, \\
\mathbb{E}_{n}=\left[(2 \sqrt{13}-5 \sqrt{2}) p_{2}+17 \sqrt{2}+2 \sqrt{13}\right] n+2(5 \sqrt{2}-2 \sqrt{13}) p_{2}-10 \sqrt{2}, \\
\mathbb{E}_{n}^{r e d}=\left[(2 \sqrt{5}-3 \sqrt{2}) p_{2}+11 \sqrt{2}+2 \sqrt{5}\right] n+(6 \sqrt{2}-4 \sqrt{5}) p_{2}-8 \sqrt{2},
\end{gathered}
$$




$$
\begin{array}{r}
\mathbb{E}_{n}^{a v r}=\left[2\left(p_{2}+1\right) \sqrt{2 \bar{d}^{2}-10 \bar{d}+13}+\sqrt{2}\left(13-p_{2}-4 \bar{d}\right)\right] n \\
-4 p_{2} \sqrt{2 \bar{d}^{2}-10 \bar{d}+13}+2 \sqrt{2}\left(p_{2}+5 \bar{d}-13\right), \text { where } \bar{d}=\frac{8 n-2}{3 n} .
\end{array}
$$

Proof. From the structure of the phenylene chain, it is easy to see that there exists only $(2,2)$, $(2,3)$ and $(3,3)$-type of edges. From Proposition 2.1, when $n=2, \mathbb{E}_{2}^{a}=4 \sqrt{2 a^{2}-10 a+13}+$ $6 \sqrt{2} \cdot|2-a|+4 \sqrt{2} \cdot|3-a|$. For $n \geq 3$, there are three possibilities to be considered (see Figure 2).

Case 1. $R P H_{n-1} \rightarrow R P H_{n}^{1}$.

$$
\begin{aligned}
& m_{22}\left(R P H_{n}^{1}\right)=m_{22}\left(R P H_{n-1}\right)+1 ; \\
& m_{23}\left(R P H_{n}^{1}\right)=m_{23}\left(R P H_{n-1}\right)+2 ; \\
& m_{33}\left(R P H_{n}^{1}\right)=m_{33}\left(R P H_{n-1}\right)+5 .
\end{aligned}
$$

Thus, $S O_{a}\left(R P H_{n}^{1}\right)=S O_{a}\left(R P H_{n-1}\right)+2 \sqrt{2 a^{2}-10 a+13}+\sqrt{2} \cdot|2-a|+5 \sqrt{2} \cdot|3-a|$.

Case 2. $R P H_{n-1} \rightarrow R P H_{n}^{2}$.

$$
\begin{aligned}
& m_{22}\left(R P H_{n}^{2}\right)=m_{22}\left(R P H_{n-1}\right)+0 \\
& m_{23}\left(R P H_{n}^{2}\right)=m_{23}\left(R P H_{n-1}\right)+4 \\
& m_{33}\left(R P H_{n}^{2}\right)=m_{33}\left(R P H_{n-1}\right)+4 .
\end{aligned}
$$

Thus, $S O_{a}\left(R P H_{n}^{2}\right)=S O_{a}\left(R P H_{n-1}\right)+4 \sqrt{2 a^{2}-10 a+13}+4 \sqrt{2} \cdot|3-a|$.

Case 3. $R P H_{n-1} \rightarrow R P H_{n}^{3}$.

$$
\begin{aligned}
& m_{22}\left(R P H_{n}^{3}\right)=m_{22}\left(R P H_{n-1}\right)+1 ; \\
& m_{23}\left(R P H_{n}^{3}\right)=m_{23}\left(R P H_{n-1}\right)+2 ; \\
& m_{33}\left(R P H_{n}^{3}\right)=m_{33}\left(R P H_{n-1}\right)+5 .
\end{aligned}
$$

Thus, $S O_{a}\left(R P H_{n}^{3}\right)=S O_{a}\left(R P H_{n-1}\right)+2 \sqrt{2 a^{2}-10 a+13}+\sqrt{2} \cdot|2-a|+5 \sqrt{2} \cdot|3-a|$.

Therefore, $\mathbb{E}_{n}^{a}=p_{1} \cdot S O_{a}\left(R P H_{n}^{1}\right)+p_{2} \cdot S O_{a}\left(R P H_{n}^{2}\right)+\left(1-p_{1}-p_{2}\right) \cdot S O_{a}\left(R P H_{n}^{3}\right)=$ $S O_{a}\left(R P H_{n-1}\right)+2\left(p_{2}+1\right) \sqrt{2 a^{2}-10 a+13}+\sqrt{2}\left(1-p_{2}\right) \cdot|2-a|+\sqrt{2}\left(5-p_{2}\right) \cdot|3-a|$. Since $E\left[\mathbb{E}_{n}^{a}\right]=\mathbb{E}_{n}^{a}$, we have

$$
\mathbb{E}_{n}^{a}=\mathbb{E}_{n-1}^{a}+2\left(p_{2}+1\right) \sqrt{2 a^{2}-10 a+13}+\sqrt{2}\left(1-p_{2}\right) \cdot|2-a|+\sqrt{2}\left(5-p_{2}\right) \cdot|3-a|
$$


After solving the recurrence relation (12) with initial condition, we get (8).

When $a=0$, we have (9). When $a=1$, we have (10). Since $\left|V\left(R P H\left(n ; p_{1}, p_{2}\right)\right)\right|=6 n$, $\left|E\left(R P H\left(n ; p_{1}, p_{2}\right)\right)\right|=8 n-2$, we have $2<\bar{d}=\frac{8 n-2}{3 n}<3$. For given $n, \bar{d}$ is a constant and therefore we get (11).

Let $\mathbb{R}_{n}=R P H\left(n ; p_{1}, 0\right)$, where $p_{1} \in[0,1]$ and $\mathbb{P}_{n}=R P H(n ; 0,1)$. By Theorem 2.2 , we have

Corollary 2.7. The Sombor indices of $\mathbb{R}_{n}$ and $\mathbb{P}_{n}$ are

$$
\begin{aligned}
& S O_{a}\left(\mathbb{R}_{n}\right)=2 n \sqrt{2 a^{2}-10 a+13}+\sqrt{2}(n+4) \cdot|2-a|+\sqrt{2}(5 n-6) \cdot|3-a|, \\
& S O_{a}\left(\mathbb{P}_{n}\right)=4(n-1) \sqrt{2 a^{2}-10 a+13}+6 \sqrt{2} \cdot|2-a|+4 \sqrt{2}(n-1) \cdot|3-a| .
\end{aligned}
$$

Corollary 2.8. Among all random phenylene chains $R P H_{n}(n \geq 2)$, we have

(1) $(17 \sqrt{2}+2 \sqrt{13}) n-10 \sqrt{2} \leq S O\left(R P H_{n}\right) \leq(12 \sqrt{2}+4 \sqrt{13}) n-4 \sqrt{13}$, with left equality iff $G \cong \mathbb{R}_{n}$, right equality iff $G \cong \mathbb{P}_{n}$.

(2) $(11 \sqrt{2}+2 \sqrt{5}) n-8 \sqrt{2} \leq S O_{\text {red }}\left(R P H_{n}\right) \leq(8 \sqrt{2}+4 \sqrt{5}) n-2 \sqrt{2}-4 \sqrt{5}$, with left equality iff $G \cong \mathbb{R}_{n}$, right equality iff $G \cong \mathbb{P}_{n}$.

(3) $2 n \sqrt{2 \bar{d}^{2}-10 \bar{d}+13}+\sqrt{2}[(13-4 \bar{d}) n+2(5 \bar{d}-13)] \leq S O_{\text {avr }}\left(R P H_{n}\right) \leq 4(n-1) \sqrt{2 \bar{d}^{2}-10 \bar{d}+13}+$ $2 \sqrt{2}[2 n(3-\bar{d})+5 \bar{d}-12]$, where $\bar{d}=\frac{8 n-2}{3 n}$, with left equality iff $G \cong \mathbb{R}_{n}$, right equality iff $G \cong \mathbb{P}_{n}$.

Proof. Since $\mathbb{E}_{n}=(n-2)(2 \sqrt{13}-5 \sqrt{2}) p_{2}+\sqrt{2}(17 n-10)+2 \sqrt{13} n$ and $(n-2)(2 \sqrt{13}-5 \sqrt{2}) \geq 0$, $S O\left(\mathrm{RPH}_{n}\right)$ reaches the maximum value when $p_{2}=1$ and reaches the minimum value when $p_{2}=0$.

Since $\mathbb{E}_{n}^{\text {red }}=(n-2)(2 \sqrt{5}-3 \sqrt{2}) p_{2}+\sqrt{2}(11 n-8)+2 \sqrt{5} n$ and $(n-2)(2 \sqrt{5}-3 \sqrt{2}) \geq 0$, $S O_{\text {red }}\left(R P H_{n}\right)$ reaches the maximum value when $p_{2}=1$ and reaches the minimum value when $p_{2}=0$.

$$
\mathbb{E}_{n}^{a v r}=(n-2)\left[2 \sqrt{2 \bar{d}^{2}-10 \bar{d}+13}-\sqrt{2}\right] p_{2}+2 n \sqrt{2 \bar{d}^{2}-10 \bar{d}+13}+\sqrt{2}[(13-4 \bar{d}) n+2(5 \bar{d}-13)]
$$

can be regarded as a linear function of $p_{2}$. Since $n \geq 2,2 \bar{d}^{2}-10 \bar{d}+13=2\left(\bar{d}-\frac{5}{2}\right)^{2}+\frac{1}{2} \geq \frac{1}{2}$, we have $2 \sqrt{2 \bar{d}^{2}-10 \bar{d}+13}-\sqrt{2} \geq 0$. Thus $S O_{a v r}\left(R P H_{n}\right)$ reaches the maximum value when $p_{2}=1$ and reaches the minimum value when $p_{2}=0$.

Denote by $\mathcal{P C}_{n}$ the set of all phenylene chains with $n$ hexagons. The average value of Sombor indices among $\mathcal{P C}_{n}$ can be characterized as

$$
A_{a}\left(\mathcal{P C}_{n}\right)=\frac{1}{\mid \mathcal{P \mathcal { C } _ { n } |}} \sum_{G \in \mathcal{P C} \mathcal{C}_{n}} S O_{a}(G)
$$


Since each element in $\mathcal{P C}_{n}$ has the same probability of occurrence, we have $p_{1}=p_{2}=1-p_{1}-$ $p_{2}=\frac{1}{3}$. Then we have the following theorem.

Theorem 2.9. The average values of Sombor indices among $\mathcal{P C}$ are

$$
A_{a}\left(\mathcal{P C}_{n}\right)=\frac{4}{3}(2 n-1) \sqrt{2 a^{2}-10 a+13}+\frac{2}{3} \sqrt{2}(n+7) \cdot|2-a|+\frac{2}{3} \sqrt{2}(7 n-8) \cdot|3-a| .
$$

\subsection{Comparisons between Sombor indices with respect to random hexagonal chains and random phenylene chains}

With the help of Theorems 2.2 and 2.6, we make a comparison between the expected values for Sombor index, reduced Sombor index and average Sombor index of a random hexagonal chain or a random phenylene chain with the same probabilities $p_{i}(i=1,2)$ (see Figure 3, 4).

Theorem 2.10. Let $H X G\left(n ; p_{1}, p_{2}\right)$ be the hexagonal chain of length $n(\geq 2)$ and $R P H\left(n ; p_{1}, p_{2}\right)$ be the random phenylene chain of length $n(\geq 2)$. Then

$$
\begin{gathered}
E[S O(G)]>E\left[S O_{\text {red }}(G)\right]>E\left[S O_{\text {avr }}(G)\right] \text {, where } G \cong H X G\left(n ; p_{1}, p_{2}\right) \text { or } \operatorname{RPH}\left(n ; p_{1}, p_{2}\right), \\
E\left[S O\left(R P H\left(n ; p_{1}, p_{2}\right)\right)\right]>E\left[S O\left(H X G\left(n ; p_{1}, p_{2}\right)\right)\right], \\
E\left[S O_{r e d}\left(R P H\left(n ; p_{1}, p_{2}\right)\right)\right]>E\left[S O_{\text {red }}\left(H X G\left(n ; p_{1}, p_{2}\right)\right)\right], \\
E\left[S O_{\text {avr }}\left(\operatorname{RPH}\left(n ; p_{1}, p_{2}\right)\right)\right]>E\left[S O_{\text {avr }}\left(H X G\left(n ; p_{1}, p_{2}\right)\right)\right] .
\end{gathered}
$$

Proof. Since $2 \leq d_{i}, d_{j} \leq 3,2<\bar{d}<3$, we have

$$
\sqrt{d_{i}^{2}+d_{j}^{2}}>\sqrt{\left(d_{i}-1\right)^{2}+\left(d_{j}-1\right)^{2}}>\sqrt{\left(d_{i}-\bar{d}\right)^{2}+\left(d_{j}-\bar{d}\right)^{2}}
$$

thus $E[S O(G)]>E\left[S O_{\text {red }}(G)\right]>E\left[S O_{\text {avr }}(G)\right]$.

Since

$$
\begin{gathered}
E\left[S O\left(R P H\left(n ; p_{1}, p_{2}\right)\right)\right]-E\left[S O\left(H X G\left(n ; p_{1}, p_{2}\right)\right)\right]=9 \sqrt{2}(n-1)>0, \\
E\left[S O_{r e d}\left(R P H\left(n ; p_{1}, p_{2}\right)\right)\right]-E\left[S O_{r e d}\left(H X G\left(n ; p_{1}, p_{2}\right)\right)\right]=6 \sqrt{2}(n-1)>0,
\end{gathered}
$$

we have

$$
\begin{gathered}
E\left[S O\left(R P H\left(n ; p_{1}, p_{2}\right)\right)\right]>E\left[S O\left(H X G\left(n ; p_{1}, p_{2}\right)\right)\right] \\
E\left[S O_{r e d}\left(R P H\left(n ; p_{1}, p_{2}\right)\right)\right]>E\left[S O_{r e d}\left(H X G\left(n ; p_{1}, p_{2}\right)\right)\right] .
\end{gathered}
$$


When $n=2$, from Theorem 2.2 and Theorem 2.6, we have $E\left[S O_{\text {avr }}\left(R P H\left(2 ; p_{1}, p_{2}\right)\right)\right]>$ $E\left[S O_{a v r}\left(H X G\left(2 ; p_{1}, p_{2}\right)\right)\right]$. Let $\bar{d}_{1}=\bar{d}\left(H X G\left(n ; p_{1}, p_{2}\right)\right)$ and $\bar{d}_{2}=\bar{d}\left(R P H\left(n ; p_{1}, p_{2}\right)\right)$. Since

$$
\frac{11}{5} \leq \bar{d}_{1}=\frac{5 n+1}{2 n+1}<\frac{5}{2}, \quad \frac{7}{3} \leq \bar{d}_{2}=\frac{8 n-2}{3 n}<\frac{8}{3}
$$

we have $\bar{d}_{1}-4 \bar{d}_{2} \geq \frac{11}{5}-4 \cdot \frac{8}{3}=-\frac{127}{15}$. Let $f(a)=2 \sqrt{2 a^{2}-10 a+13}$, then $f\left(\bar{d}_{2}\right)-f\left(\bar{d}_{1}\right) \geq$ $f\left(\frac{5}{2}\right)-f\left(\frac{11}{5}\right)=\sqrt{2}-\frac{2}{5} \sqrt{17}$. By $(7)$ and $(12)$,

$$
\begin{aligned}
& \mathbb{E}_{n}^{a v r}-\mathbb{E}_{n-1}^{a v r}-\left(E_{n}^{a v r}-E_{n-1}^{a v r}\right) \\
= & \left(p_{2}+1\right)\left(2 \sqrt{2 \bar{d}_{2}^{2}-10 \bar{d}_{2}+13}-2 \sqrt{2 \bar{d}_{1}^{2}-10 \bar{d}_{1}+13}\right)+\sqrt{2}\left(9+\bar{d}_{1}-4 \bar{d}_{2}\right) \\
\geq & \left(p_{2}+1\right) \cdot\left(\sqrt{2}-\frac{2}{5} \sqrt{17}\right)+\sqrt{2} \cdot\left(9-\frac{127}{15}\right) \\
\geq & 2\left(\sqrt{2}-\frac{2}{5} \sqrt{17}\right)+\sqrt{2} \cdot\left(9-\frac{127}{15}\right) \\
= & \left(11-\frac{127}{15}\right) \sqrt{2}-\frac{7}{5} \sqrt{17}>0 .
\end{aligned}
$$

Therefore, $E\left[S O_{\text {avr }}\left(R P H\left(n ; p_{1}, p_{2}\right)\right)\right]>E\left[S O_{\text {avr }}\left(H X G\left(n ; p_{1}, p_{2}\right)\right)\right]$.

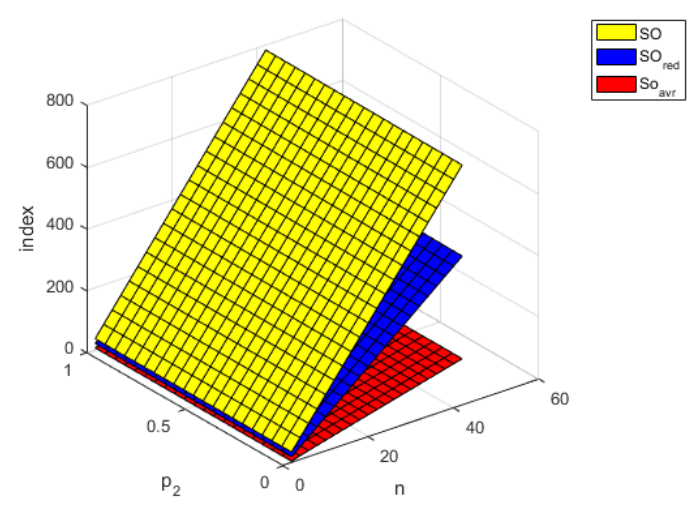

Figure 3: Difference between $S O\left(H X G_{n}\right)$, $S O_{\text {red }}\left(H X G_{n}\right)$ and $S O_{a v r}\left(H X G_{n}\right)$

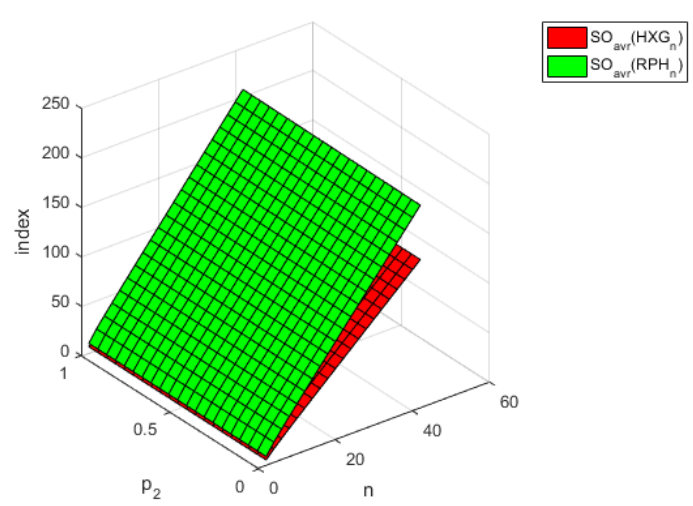

Figure 4: Difference between $S O_{\text {avr }}\left(H X G_{n}\right)$ and $S O_{\text {avr }}\left(R P H_{n}\right)$

\section{The Sombor indices of graphene, coronoid systems and carbon nanocones}

Topological indices are important graph invariants used for describing various properties of molecules. Methods for computing topological indices of some molecular graphs such as benzenoid systems, phenylenes or coronoid systems were studied in [6, 33, 37]. In this section, we study the Sombor indices of graphene, coronoid systems and carbon nanocones. 
Graphene [5,26], denoted by $G N(n, k)$, is a flat monolayer of carbon atoms tightly packed into a two-dimensional hexagonal lattice that forms a basic building block for graphitic materials of different forms (see Figure 5). Due to the $\mathrm{C}-\mathrm{C}$ covalent bonds, graphene is the hardest material known in nature [13]. There are various results about the topological indices of graphene in recent years [1,3].

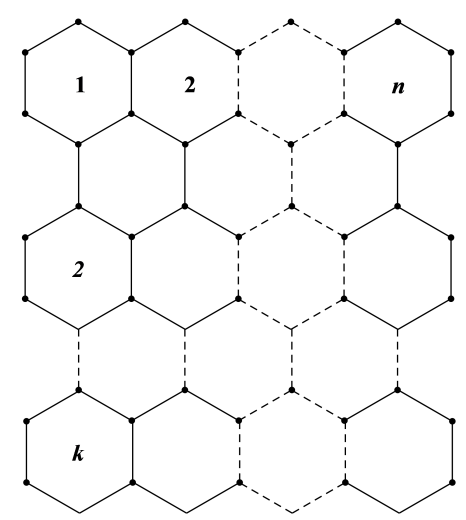

Figure 5: Structure of graphene $G N(n, k)$.

Theorem 3.1. Let $G$ be the graphene $G N(n, k), 1 \leq k \leq n$. Then

$$
\begin{gathered}
S O(G)=4 \sqrt{13}(n+k-2)+\sqrt{2}(18 n k-15 n-11 k+20), \\
S O_{r e d}(G)=4 \sqrt{5}(n+k-2)+\sqrt{2}(12 n k-8 k-10 n+12), \\
S O_{a v r}(G)=\frac{4(k+n-2)}{k(2 n+1)} \sqrt{4 k^{2} n^{2}-4 k^{2} n+5 k^{2}-4 k n^{2}+6 k n+2 n^{2}} \\
+\frac{\sqrt{2}\left(16 k^{2} n-12 k^{2}+6 k n^{2}-9 k n+4 k-5 n^{2}\right)}{k(2 n+1)} .
\end{gathered}
$$

Proof. From the structure of graphene $G N(n, k)$, it is easy to see that there exists only $(2,2)$, $(2,3)$ and $(3,3)$-type of edges. Since $m_{22}(G)=2 k+4, m_{23}(G)=4 n+4 k-8, m_{33}(G)=$ $6 n k-5 k-5 n+4$, from Proposition 2.1, we have

$$
S O_{a}(G)=4(n+k-2) \sqrt{2 a^{2}-10 a+13}+2 \sqrt{2}(k+2) \cdot|2-a|+\sqrt{2}(6 n k-5 k-5 n+4) \cdot|3-a| .
$$

Thus

$$
\begin{aligned}
& S O(G)=4 \sqrt{13}(n+k-2)+\sqrt{2}(18 n k-15 n-11 k+20), \\
& S O_{r e d}(G)=4 \sqrt{5}(n+k-2)+\sqrt{2}(12 n k-8 k-10 n+12) .
\end{aligned}
$$

Since $|V(G)|=2(2 n+1) k,|E(G)|=(6 n+1) k-n$, we have $2<\bar{d}=\frac{(6 n+1) k-n}{(2 n+1) k}<3$. Thus

$$
S O_{a v r}(G)=\frac{4(k+n-2)}{k(2 n+1)} \sqrt{4 k^{2} n^{2}-4 k^{2} n+5 k^{2}-4 k n^{2}+6 k n+2 n^{2}}
$$




$$
+\frac{\sqrt{2}\left(16 k^{2} n-12 k^{2}+6 k n^{2}-9 k n+4 k-5 n^{2}\right)}{k(2 n+1)} .
$$

The proof is completed.

A coronoid system can be regarded as a benzenoid system that is allowed to have 'holes' such that the perimeter of the coronoid system and the perimeters of the holes are pairwise disjoint. There are many results on topological index of coronoid systems [9, 18]. We now consider a special family of coronoid systems, denoted by $K(n, p, r)$ (see Figure 6), which is formally generated from polycyclic benzenoid systems by circumcising some interior atoms or bonds.

Theorem 3.2. Let $G$ be the $K(n, p, r)$ coronoid structure with $r \geq 1, n \geq 3$ and $1 \leq p \leq n$. Then

$$
\begin{gathered}
S O(G)=4 \sqrt{13}(2 n+4 p+3 r-6)+3 \sqrt{2}\left[3(3 r-2)(2 p+n)+9 r^{2}-15 r+16\right], \\
S O_{r e d}(G)=4 \sqrt{5}(2 n+4 p+3 r-6)+\sqrt{2}\left[4(3 r-2)(2 p+n)+18 r^{2}-30 r+30\right], \\
S O_{a v r}(G)=\frac{1}{r+1}\left[4 \sqrt{r^{2}+1}(2 n+4 p+3 r-6)+\sqrt{2}(1+6 r)\right] .
\end{gathered}
$$

Proof. From the structure of $K(n, p, r)$ coronoid structure, it is easy to see that there exists only $(2,2),(2,3)$ and $(3,3)$-type of edges. Since $m_{22}(G)=6, m_{23}(G)=8(2 p+n)+12(r-2)$, $m_{33}(G)=2(3 r-2)(2 p+n)+3\left(3 r^{2}-5 r+4\right)$, from Proposition 2.1, we have

$S O_{a}(G)=4 \sqrt{2 a^{2}-10 a+13}(4 p+2 n+3 r-6)+6 \sqrt{2} \cdot|2-a|+|3-a| \cdot \sqrt{2}\left[2(3 r-2)(2 p+n)+3\left(3 r^{2}-5 r+4\right)\right]$.

Thus

$$
\begin{gathered}
S O(G)=4 \sqrt{13}(2 n+4 p+3 r-6)+3 \sqrt{2}\left[3(3 r-2)(2 p+n)+9 r^{2}-15 r+16\right], \\
S O_{r e d}(G)=4 \sqrt{5}(4 p+2 n+3 r-6)+\sqrt{2}\left[4(3 r-2)(n+2 p)+18 r^{2}-30 r+30\right] .
\end{gathered}
$$

Since $|V(G)|=2(r+1)(4 p+2 n+3 r-3),|E(G)|=(3 r+2)(4 p+2 n+3 r-3)$, we have $2<\bar{d}=\frac{3 r+2}{r+1}<3$. Thus

$$
S O_{a v r}(G)=\frac{1}{r+1}\left[4 \sqrt{r^{2}+1}(2 n+4 p+3 r-6)+\sqrt{2}(1+6 r)\right] .
$$

The proof is completed.

From Theorem 3.2 , it is easy to obtain Corollary 3.3 and Corollary 3.4 as special cases. More precisely, we use the previous theorem on $K(2,1, r)$ and $K(2,2, r)$ to compute the indices for $r$-circumscribed $\mathrm{C}_{32} \mathrm{H}_{16}$ and $\mathrm{C}_{48} \mathrm{H}_{24}$ coronoid structures. 


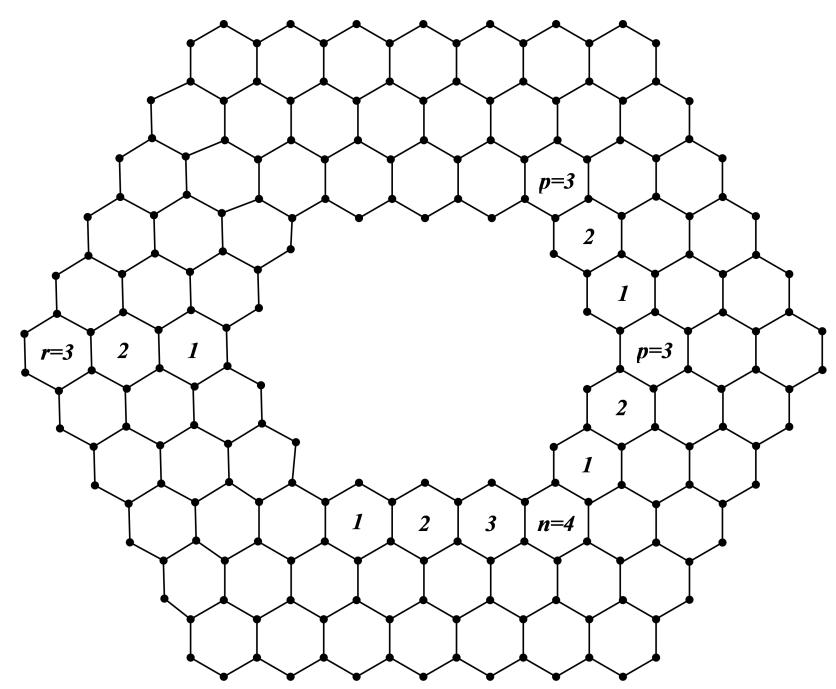

Figure 6: Structure of coronoid system $K(n, p, r)$.

Corollary 3.3. Let $G$ be an $r$-circumscribed $C_{32} H_{16}$ coronoid structure $(r \geq 1)$. Then

$$
\begin{gathered}
S O(G)=4 \sqrt{13}(2+3 r)+3 \sqrt{2}\left(9 r^{2}+21 r-8\right), \\
S O_{r e d}(G)=4 \sqrt{5}(2+3 r)+2 \sqrt{2}\left(9 r^{2}+9 r-1\right), \\
S O_{a v r}(G)=\frac{1}{r+1}\left[4 \sqrt{r^{2}+1}(2+3 r)+\sqrt{2}(1+6 r)\right] .
\end{gathered}
$$

Corollary 3.4. Let $G$ be an $r$-circumscribed $C_{48} H_{24}$ coronoid structure $(r \geq 1)$. Then

$$
\begin{gathered}
S O(G)=12 \sqrt{13}(2+r)+3 \sqrt{2}\left(9 r^{2}+39 r-20\right), \\
S O_{r e d}(G)=12 \sqrt{5}(2+r)+6 \sqrt{2}\left(3 r^{2}+7 r-3\right), \\
S O_{a v r}(G)=\frac{1}{r+1}\left[12 \sqrt{r^{2}+1}(2+r)+\sqrt{2}(1+6 r)\right] .
\end{gathered}
$$

Carbon nanocones, denoted by $C N C_{k}(n)$, are conical structures, which are conceived as curved forms of graphite sheet obtained by excising a wedge and subsequently joining the edges (see Figure 7). Carbon nanocones have a wide range of applications, such as caping ultrafine gold needles, which attracted the attention of both theoretical and experimental chemists. There are many results on topological index of carbon nanocones [2,25].

Theorem 3.5. Let $G$ be the carbon nanocone structure $C N C_{k}(n)$ with $k>4$ and $n \geq 1$. Then

$$
\begin{aligned}
& S O(G)=2 \sqrt{13} k n+\frac{\sqrt{2} k}{2}\left(9 n^{2}+3 n+4\right), \\
& S O_{r e d}(G)=2 \sqrt{5} k n+\sqrt{2} k\left(3 n^{2}+n+1\right), \\
& S O_{a v r}(G)=\frac{k n}{2}\left(\frac{4}{n+1} \sqrt{n^{2}+1}+3 \sqrt{2}\right) .
\end{aligned}
$$




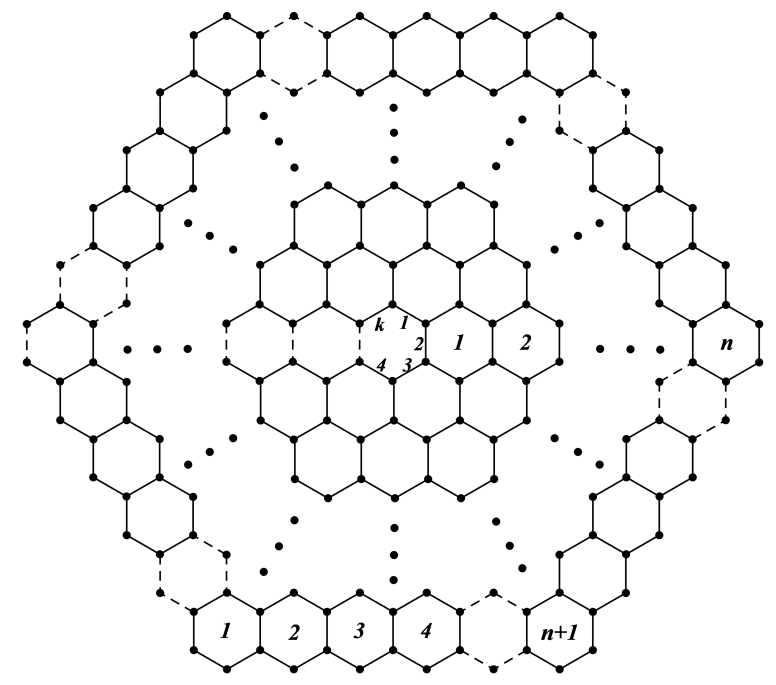

Figure 7: Structure of carbon nanocone $C N C_{k}(n)$.

Proof. From the structure of carbon nanocone $C N C_{k}(n)$, it is easy to see that there exists only $(2,2),(2,3)$ and $(3,3)$-type of edges. Since $m_{22}(G)=k, m_{23}(G)=2 k n, m_{33}(G)=k n(3 n+1) / 2$, from Proposition 2.1, we have

$$
S O_{a}(G)=2 k n \sqrt{2 a^{2}-10 a+13}+\frac{\sqrt{2} k}{2}[2 \cdot|2-a|+|3-a| \cdot(3 n+1) n] .
$$

Thus,

$$
\begin{aligned}
& S O(G)=2 \sqrt{13} k n+\frac{\sqrt{2} k}{2}\left(9 n^{2}+3 n+4\right), \\
& S O_{\text {red }}(G)=2 \sqrt{5} k n+\sqrt{2} k\left(3 n^{2}+n+1\right) .
\end{aligned}
$$

Since $|V(G)|=k(n+1)^{2},|E(G)|=k(n+1)(3 n+2) / 2$, we have $2<\bar{d}=\frac{3 n+2}{n+1}<3$. Thus

$$
S O_{a v r}(G)=\frac{k n}{2}\left(\frac{4}{n+1} \sqrt{n^{2}+1}+3 \sqrt{2}\right) .
$$

The proof is completed.

Corollary 3.6. Let $G$ be n-circumscribed one pentagonal carbon nanocone structure $\mathrm{CNC}_{5}(n)$ with $n \geq 1$. Then

$$
\begin{aligned}
& S O(G)=10 \sqrt{13} n+\frac{5 \sqrt{2}}{2}\left(9 n^{2}+3 n+4\right), \\
& S O_{\text {red }}(G)=10 \sqrt{5} n+5 \sqrt{2}\left(3 n^{2}+n+1\right), \\
& S O_{\text {avr }}(G)=\frac{5 n}{2}\left(\frac{4}{n+1} \sqrt{n^{2}+1}+3 \sqrt{2}\right) .
\end{aligned}
$$

It's clear that $E[S O(G)]>E\left[S O_{\text {red }}(G)\right]>E\left[S O_{\text {avr }}(G)\right]$ for graph $G$ with only $(2,2)$, $(2,3)$ and $(3,3)$-type of edges. The graphical profiles of the comparison between Sombor, reduced Sombor and average Sombor indices of graphene $G N(n, k)$ or carbon nanocone structure 


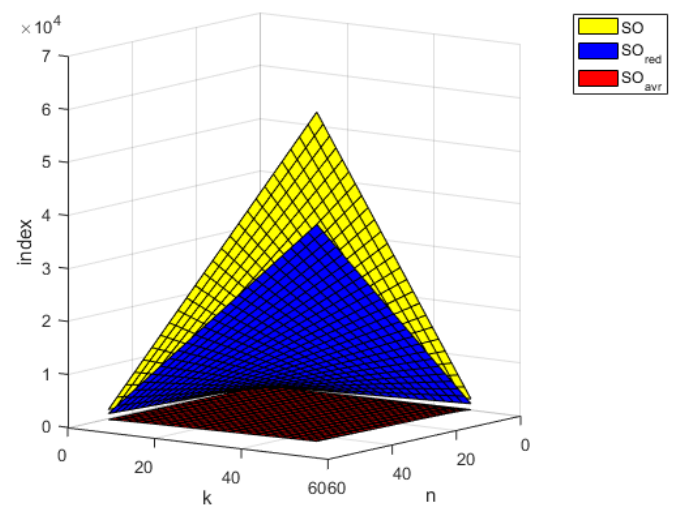

Figure 8: Differences between Sombor, reduced Sombor and average Sombor indices of $G N(n, k)$.

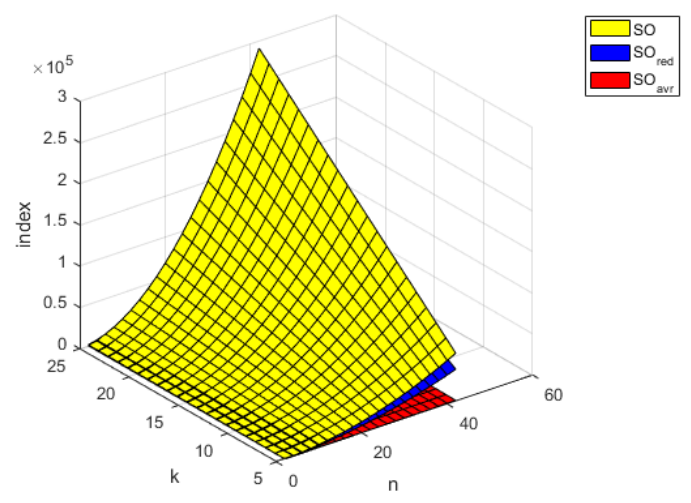

Figure 9: Differences between Sombor, reduced Sombor and average Sombor indices of $C N C_{k}(n)$.

\begin{tabular}{llll}
\hline$(n, p, r)$ & $S O(G)$ & $S O_{\text {red }}(G)$ & $S O_{\text {avr }}(G)$ \\
\hline$(3,1,1)$ & 207.02 & 116.35 & 24.75 \\
$(3,1,2)$ & 492.12 & 261.98 & 35.94 \\
$(3,1,3)$ & 853.58 & 458.51 & 47.83 \\
$(4,2,4)$ & 1759.79 & 929.34 & 79.64 \\
$(4,2,5)$ & 2388.54 & 1278.61 & 92.29 \\
$(4,2,6)$ & 3093.66 & 1678.80 & 104.80 \\
$(5,2,1)$ & 373.31 & 210.53 & 47.38 \\
$(5,3,2)$ & 970.66 & 505.07 & 71.72 \\
$(5,4,3)$ & 1797.10 & 918.41 & 98.42 \\
$(6,4,4)$ & 2696.53 & 1376.08 & 119.22 \\
$(6,4,5)$ & 3554.38 & 1827.18 & 133.08 \\
$(6,4,6)$ & 4488.60 & 2329.18 & 146.51 \\
$(9,5,7)$ & 6852.57 & 3508.95 & 194.98 \\
$(9,6,8)$ & 8748.16 & 4482.31 & 222.69 \\
$(9,7,9)$ & 10872.85 & 5574.47 & 250.46 \\
\hline
\end{tabular}

Table 1: Numeric differences for $K(n, p, r)$

$C N C_{k}(n)$ is give in Figure 8, 9. The numerical comparison of the Sombor indices with respect to different types of $K(n, p, r)$ coronoid structure is give in Table 1 .

\section{Conclusion}

In this paper, the expected values of Sombor index, reduced Sombor index and average Sombor index have been determined for random hexagonal chains and random phenylene chains. Explicit formulae for Sombor index, reduced Sombor index and average Sombor index of some chemical graphs such as graphene, coronoid systems and carbon nanocones are given. And detailed comparisons between these indices with respect to different chemical graphs have been 
determined explicitly. The structural characteristics of the compound can be deduced from the topological index formulae, which provides a theoretical basis for drug discovery and synthetic organic chemistry.

\section{References}

[1] M. Arockiaraj, J. Clement, K. Balasubramanian, Analytical expressions for topological properties of polycyclic benzenoid networks, J. Chemometr. 30 (2016) 682-697.

[2] M. Arockiaraj, J. Clement, N. Tratnik, Mostar indices of carbon nanostructures and circumscribed donut benzenoid systems, Int. J. Quantum Chem. 119 (2019) \#e26043.

[3] M. Arockiaraj, J. Clement, N. Tratnik, S. Mushtaq, K. Balasubramanian, Weighted Mostar indices as measures of molecular peripheral shapes with applications to graphene, graphyne and graphdiyne nanoribbons, SAR QSAR Environ. Res. 31 (2020) 187-208.

[4] S. Alikhani, N. Ghanbari, Sombor index of polymers, MATCH Commun. Math. Comput. Chem. 86 (2021) 715-728.

[5] H.P. Boehm, R. Setton, E. Stumpp, Nomenclature and terminology of graphite intercalation compounds, Carbon 24 (1986) 241-245.

[6] S. Brezovnik, N. Tratnik, General cut method for computing Szeged-like topological indices with applications to molecular graphs, Int. J. Quantum Chem. 121 (2021) \#e26530.

[7] R. Cruz, I. Gutman, J. Rada, Sombor index of chemical graphs, Appl. Math. Comput. 399 (2021) \#126018.

[8] R. Cruz, J. Rada, Extremal values of the Sombor index in unicyclic and bicyclic graphs, $J$. Math. Chem. 59 (2021) 1098-1116.

[9] R. Cruz, A.D. Santamaría-Galvis, J. Rada, Extremal values of vertex-degree-based topological indices of coronoid systems, Int. J. Quantum Chem. 121 (2021) \#e26536.

[10] K. C. Das, A. S. Cevik, I. N. Cangul, Y. Shang, On Sombor index, Symmetry 13 (2021) \#140.

[11] K. C. Das, I. Gutman, On Sombor index of trees, submitted. 
[12] H. Deng, Z. Tang, R. Wu, Molecular trees with extremal values of Sombor indices, Int. J. Quantum Chem. 121 (2021) \# 26622.

[13] A.K. Geim, K.S. Novoselov, The rise of graphene, Nat. Mater. 6 (2007) 183-191.

[14] I. Gutman, Geometric approach to degree-based topological indices: Sombor indices, MATCH Commun. Math. Comput. Chem. 86 (2021) 11-16.

[15] I. Gutman, Some basic properties of Sombor indices, Open J. Discret. Appl. Math. 4 (2021) $1-3$.

[16] B. Horoldagva, C. Xu, On Sombor index of graphs, MATCH Commun. Math. Comput. Chem. 86 (2021) 703-713.

[17] A. Jahanbani, The expected values of the first Zagreb and Randić indices in random polyphenyl chains, Polycyclic Aromat. Compd. (2020) doi: 10.1080/10406638.2020.1809472.

[18] K. Julietraja, P. Venugopal, Computation of degree-based topological descriptors using Mpolynomial for coronoid systems, Polycyclic Aromat. Compd. (2020) doi: 10.1080/10406638. 2020.1804415 .

[19] V.R. Kulli, I. Gutman, Computation of Sombor indices of certain networks, International Journal of Applied Chemistry, 8 (2021) 1-5.

[20] H. Liu, H. Chen, Q. Xiao, X. Fang, Z. Tang, More on Sombor indices of chemical graphs and their applications to the boiling point of benzenoid hydrocarbons, Int. J. Quantum Chem. (2021), doi: 10.1002/qua.26689.

[21] H. Liu, L. You, Y. Huang, Ordering chemical graphs by Sombor indices and its applications, MATCH Commun. Math. Comput. Chem. 87 (2022) in press.

[22] H. Liu, L. You, Z. Tang, J.B. Liu, On the reduced Sombor index and its applications, MATCH Commun. Math. Comput. Chem. 86 (2021) 729-753.

[23] H. Liu, M. Zeng, H. Deng, Z. Tang, Some indices in the random spiro chains, Iranian J. Math. Chem. 11 (2020) 255-270.

[24] I. Milovanović, E. Milovanović, M. Matejić, On some mathematical properties of Sombor indices, Bull. Int. Math. Virtual Inst. 11 (2021) 341-353. 
[25] W. Nazeer, A. Farooq, M. Younas, M. Munir, S.M. Kang, On molecular descriptors of carbon nanocones, Biomolecules 8 (2018) 92-103.

[26] K.S. Novoselov, A.K. Geim, S.V. Morozov, D. Jiang, Y. Zhang, S.V. Dubonos, A.A. Firsov, Electric field in atomically thin carbon films, Science 306 (2004) 666-669.

[27] Z. Raza, The harmonic and second Zagreb indices in random polyphenyl and spiro chains, Polycyclic Aromat. Compd. (2020) doi:10.1080/10406638.2020.1749089.

[28] Z. Raza, Zagreb connection indices for some benzenoid systems, Polycyclic Aromat. Compd. (2020) doi:10.1080/10406638.2020.1809469.

[29] Z. Raza, The expected values of arithmetic bond connectivity and geometric indices in random phenylene chains, Heliyon 6 (2020) \#e04479.

[30] Z. Raza, The expected values of some indices in random phenylene chains, Eur. Phys. J. Plus 136 (2021) \#91.

[31] I. Redžepović, Chemical applicability of Sombor indices, J. Serb. Chem. Soc. (2021) doi:10.2298/JSC201215006R.

[32] T. Réti, T. Došlić, A. Ali, On the Sombor index of graphs, Contrib. Math. 3 (2021) 11-18.

[33] N. Tratnik, Computing weighted Szeged and PI indices from quotient graphs, Int. J. Quantum Chem. 119 (2019) \# 26006.

[34] N. Trinajstic, Chemical graph theory, Routledge, 2018.

[35] Z. Wang, Y. Mao, Y. Li, B. Furtula, On relations between Sombor and other degree-based indices, J. Appl. Math. Comput. (2021) doi:10.1007/s12190-021-01516-x.

[36] W. Yang, F. Zhang, Wiener index in random polyphenyl chains, MATCH Commun. Math. Comput. Chem. 68 (2012) 371-376.

[37] P. Žigert Pleteršek, The edge-Wiener index and the edge-hyper-Wiener index of phenylenes, Discrete Appl. Math. 255 (2019) 326-333. 